PROCEEDINGS OF THE

AMERICAN MATHEMATICAL SOCIETY

Volume 42, Number 1, January 1974

\title{
REGULAR NEIGHBORHOODS AND TOPOLOGICAL MANIFOLDS
}

GLENN P. WELLER ${ }^{1}$

\begin{abstract}
This paper defines a notion of topological regular neighborhood for compact triangulable subspaces of topological manifolds, proves a uniqueness theorem for such neighborhoods, and shows that this theorem is in a sense the best possible. These results have obvious applications to regular neighborhoods in manifolds having more than one piecewise linear structure.
\end{abstract}

1. Introduction and statement of results. The study of piecewise linear (PL) manifolds uses heavily the theory of regular neighborhoods. In view of the results of Kirby, Siebenmann and others on the Hauptvermutung and triangulation problems, it is natural to ask: To what extent are regular neighborhoods unique in a manifold with more than one PL structure? The Theorem of this paper shows that they are topologically ambiently isotopic in certain circumstances. In order to state and prove the Theorem it is convenient to make the following definition, which is a reasonable analogue of the usual PL notion.

Definition. Let $X$ be a compact triangulable subspace of the interior of a topological manifold $Q$. We say that $N$ is a topological regular neighborhood of $X$ in $Q$ if:

(1) $N$ is a closed neighborhood of $X$ contained in Int $Q$ (the interior of $Q$ ).

(2) There is a PL structure on some open neighborhood of $N$ with respect to which $X$ is a PL subspace and $N$ is a regular neighborhood of $X$.

THEOREM. Let $N_{1}$ and $N_{2}$ be topological regular neighborhoods of a connected compact triangulable subspace $X^{p}$ of Int $Q^{n}$ where $n \geqq 6$ and $n \geqq p+3$. Then there is an ambient isotopy $h_{t}$ of $Q$ such that

(1) $h_{t}$ is fixed on $X$ and outside a compact set in Int $Q$,

(2) $h_{0}=1 Q$,

(3) $h_{1}\left(N_{1}\right)=N_{2}$.

Presented to the Society, January 25, 1973; received by the editors January 11, 1973. AMS (MOS) subject classifications (1970). Primary 57A15, 57C40; Secondary 57C25, 57C10.

Key words and phrases. Topological manifold, piecewise linear manifold, regular neighborhood, triangulation, Hauptvermutung, Whitehead torsion, mapping cylinder neighborhood, open cone neighborhood.

1 Partially supported by NSF Grant GP 28487.

(C) American Mathematical Society 1974 
There are two ways to attempt to strengthen the Theorem. The first is to require $h_{1} \mid N_{1}$ to be PL. A counterexample to this strengthening is given in $\S 3$. The second way is to remove the codimension three hypothesis. The necessity of this hypothesis is shown in $\$ 4$.

2. Proof of the Theorem. Since Int $N_{1}$ and Int $N_{2}$ are open mapping cylinder neighborhoods of $X$, Theorem 1 of [9] gives a homeomorphism $h$ of Int $N_{1}$ onto Int $N_{2}$ which is fixed on a neighborhood $U$ of $X$. Let $N$ be a PL regular neighborhood of $X$ in the PL manifold $N_{1}$ such that $N \subset U$. By PL regular neighborhood theory there is an isotopy of the desired type throwing $N_{1}$ onto $N$. We shall find an isotopy throwing $N$ onto $N_{2}$. The composition of these two isotopies satisfies the conclusion of the Theorem.

Let $R=N_{2} \mid$ Int $N$. Since $h$ is fixed on $U$,

$$
\begin{aligned}
R \backslash \partial N_{2} & =N_{2} \mid\left(\partial N_{2} \cup \text { Int } N\right) \\
& =h\left[N_{1} \backslash\left(\partial N_{1} \cup \text { Int } N\right)\right] \approx \partial N \times(0,1]
\end{aligned}
$$

(“ $\approx$ " means "is homeomorphic to"). Thus the composite inclusion $\partial N \rightarrow R \backslash \partial N_{2} \rightarrow R$ is a homotopy equivalence.

In particular, $H^{4}\left(R, \partial N ; Z_{2}\right)=0$. A collar neighborhood of $\partial N$ in $R$ has a PL structure agreeing with the PL structure on $\partial N$. The structure on $\partial N$ thus extends by [7] to a PL structure on $R$.

Now $N_{1} \mid N \approx \partial N_{1} \times[0, \infty)$ with $\partial N_{1}$ identified with $\partial N_{1} \times 0$. So Int $R=$ $h\left(\right.$ Int $\left.N_{1} \mid N\right) \approx \partial N_{1} \times(0, \infty)$. Let $V \approx \partial N_{2} \times(0, \infty)$ be the interior of a collar neighborhood of $\partial N_{2}$ in $R$ where $\partial N_{2} \times 0$ is identified with $\partial N_{2}$. Let (Int $R)^{*}$ and $V^{*}$ denote Int $R$ and $V$ with the infinite point in the onepoint compactification of $R \backslash \partial N_{2}$ added on. Then (Int $\left.R\right)^{*}$ and $V^{*}$ are open cone neighborhoods of the infinite point. By [8] there is a homeomorphism $k$ of $V^{*}$ onto (Int $\left.R\right)^{*}$ which is fixed near the infinite point. When the infinite point is deleted, $k$ is a homeomorphism of $V$ onto Int $R$ fixed on $\partial N_{2} \times(0, \varepsilon]$ for some $\varepsilon$. Thus

$$
\partial N_{2} \stackrel{\approx}{\longrightarrow} \partial N_{2} \times \varepsilon \longrightarrow V \stackrel{k}{\longrightarrow} \text { Int } R \stackrel{\subset}{\longrightarrow} R
$$

is a homotopy equivalence which is homotopic to the inclusion $\partial N_{2} \rightarrow R$.

So $R$ is a PL $h$-cobordism between $\partial N$ and $\partial N_{2} . N$ and $R$ are joined together along $\partial N$, a common PL submanifold, and $N \cup R=N_{2}$. Let $N_{3}$ denote $N_{2}$ with the PL structure coming from $N$ and $R$. Also let $f: N_{2} \longrightarrow N_{3}$ be a cellular homotopy equivalence which is homotopic to the identity map of $N_{2}$.

Claim 1. The Whitehead torsion $\tau\left(N_{3}, N\right)=0$. Let $X_{2}$ and $X_{3}$ denote $X$ considered as a PL subspace of the PL manifolds $N_{2}$ and $N_{3}$ respectively. The identity map $1_{X}: X_{2} \rightarrow X_{3}$ is a topological but not necessarily a PL 
homeomorphism which is homotopic to a cellular homotopy equivalence $g: X_{2} \rightarrow X_{3}$. So we have the following homotopy commutative diagram, where the vertical maps are PL inclusions:

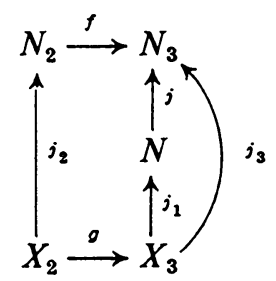

Thus, $\tau\left(j_{3} g\right)=\tau\left(f j_{2}\right)$. Applying [11, Lemma 7.8] to both sides of this equation we get

$$
\tau\left(j_{3}\right)+\left(j_{3}\right)_{*} \tau(g)=\tau(f)+f_{*} \tau\left(j_{2}\right)
$$

By [2], $\tau(f)$ and $\tau(g)$ are 0 . Since $N_{2}$ collapses to $X_{2}, \tau\left(j_{2}\right)=0$. Hence $\tau\left(j_{3}\right)=0$. But $\tau\left(j_{3}\right)=\tau\left(j j_{1}\right)=\tau(j)+j_{*} \tau\left(j_{1}\right)$. Since $N$ collapses to $X_{3}, \tau\left(j_{1}\right)=$ 0. So $\tau\left(N_{3}, N\right)=\tau(j)=\tau\left(j_{3}\right)=0$.

Claim 2. $R \approx \partial N \times I$. By the excision lemma for Whitehead torsion $\left((20.3)\right.$ of [3] expressed in the notation of [11] $j_{*} \tau(R, \partial N)=\tau\left(N_{3}, N\right)=0$ where $j: R \rightarrow N_{3}$ is the inclusion. Since the codimension of $X$ is at least three, a simple general position argument shows that $j$ induces an isomorphism of fundamental groups. Hence, $j_{*}$ is an isomorphism of the respective Whitehead groups, and it follows that $\tau(R, \partial N)=0$. So the $s$-cobordism theorem finishes the proof of the claim.

Let $\mathfrak{N}$ be a PL regular neighborhood of $X$ in the PL manifold $N$. Then since $R \approx \partial N \times I$, we have that $N \backslash$ Int $\mathfrak{N}$ and $N_{2} \backslash$ Int $\mathfrak{N}$ are collar neighborhoods of $\partial \mathfrak{N}$ in $Q \backslash$ Int $\mathfrak{N}$. By [1] there is an ambient isotopy of $Q \backslash$ Int $\mathfrak{N}$ fixed on $\partial \mathfrak{N}$ and outside a compact subset of Int $Q$ throwing $N \backslash$ Int $\mathfrak{N}$ onto $N_{2}$ ।Int $\mathfrak{R}$. The extension of this isotopy by the identity to all of $Q$ then throws $N$ onto $N_{2}$. The proof of the Theorem is thus finished.

3. A counterexample.

SuPPOSITION. In the Theorem $h_{1} \mid N_{1}$ can be chosen to be PL.

Let $n \geqq 6$ be fixed and $B(s)=\left\{y \in R^{n-3}|| y \mid \leqq s\right\}$. Let $S^{3}$ have its usual PL structure. Now $H^{3}\left(S^{3} \times R^{n-3} ; Z_{2}\right)=Z_{2}$. Let $\Re$ denote $S^{3} \times R^{n-3}$ with the PL structure which is exotic up to isotopy [6]. On the basis of the supposition an isotopy $K_{t}: S^{3} \times R^{n-3} \rightarrow \Re$ will be constructed so that $K_{0}=1$ and $K_{1}$ is a PL homeomorphism. This will contradict the exoticity of $\Re$ and disprove the supposition.

Consider $S^{3} \times 0 \subset \Re$. By [10] there is an ambient isotopy $\alpha_{t}: \Re \rightarrow \Re$ fixed outside the interior of $S^{3} \times B(1)$ such that $\alpha_{0}=1_{\Re}$ and $\Sigma=\alpha_{1}\left(S^{3} \times 0\right)$ is a PL subspace of $\Re$. 
Claim 1. If $C$ is a compact subset of $\Re$, then there is a PL regular neighborhood of $\Sigma$ in $\Re$ which contains $C$.

Assuming Claim 1 for the moment, we will construct $K_{t}$. Let $M_{i}$, $\mathfrak{M}_{i}, i \geqq 0$, be sequences of PL regular neighborhoods of $S^{3} \times 0$ in $S^{3} \times R^{n-3}$ such that $S^{3} \times B(i) \subset M_{i} \subset$ Int $\mathfrak{M}_{i} \subset \mathfrak{M}_{i} \subset M_{i+1}$. Then $\bigcup_{i \geqq 0} M_{i}=S^{3} \times R^{n-3}$. Similarly, there are sequences $N_{i}, \mathfrak{N}_{i}, i \geqq 0$, of PL regular neighborhoods of $\Sigma$ in $\Re$ such that $N_{i} \subset$ Int $\mathfrak{N}_{i} \subset \mathfrak{N}_{i} \subset N_{i+1}$ and $\bigcup_{i \geqq 0} N_{i}=\Re$. Claim 1 is necessary to construct these $N_{i}$.

We now prove by induction the following statement:

$C(k)$. For each integer $0 \leqq i<k$ there is an isotopy $h_{i, t}: S^{3} \times R^{n-3} \rightarrow \Re$ for $0 \leqq t \leqq i+1$ such that

(a) $h_{i, t}=h_{i-1, t}$ for $0 \leqq t \leqq i$,

(b) $h_{i, i+1} \mid \mathfrak{M}_{i}$ is a PL homeomorphism of the pair $\left(\mathfrak{M}_{i}, M_{i}\right)$ onto $\left(\mathfrak{N}_{i}, N_{i}\right)$.

(c) $h_{i, t}=h_{i-1 . i}$ on $M_{i-1}$ for $i \leqq t \leqq i+1$.

To establish $C(1), h_{0 . t}$ must be defined. Let $\alpha_{1} \mathfrak{M}_{0}$ have the PL structure from $\mathfrak{M}_{0}$ via $\alpha_{1}$. Since $\alpha_{1}$ is an ambient homeomorphism, $\alpha_{1} \mathfrak{M}_{0}$ is a topological regular neighborhood of $\Sigma$ in $\Re$. By the supposition, there is an ambient isotopy $h_{t}$ of $\Re$ fixed on $\Sigma$ and outside a compact set such that $h_{1} \mid \alpha_{1} \mathfrak{M}_{0}$ is a PL homeomorphism onto $\mathfrak{N}_{0}$. Now $h_{1} \alpha_{1} M_{0}$ and $N_{0}$ are PL regular neighborhoods of $\Sigma$ in $\mathfrak{N}_{0}$. So there is a PL ambient isotopy $\mu_{t}, 0 \leqq t \leqq 1$, of $\Re$ such that $\mu_{0}=1_{\Re}, \mu_{t}$ is fixed on $\Sigma$ and outside a compact subset of Int $\mathfrak{N}_{0}$, and $\mu_{1} h_{1} \alpha_{1} M_{0}=N_{0}$. Set $h_{0 . t}=\mu_{t} h_{t} \alpha_{t}$.

Suppose $C(k)$ is true. To prove $C(k+1) h_{k, t}$ must be defined. Let $h_{k-1, k}$ be denoted $g$ for simplicity. Give $g \mathfrak{M}_{k}$ the PL structure from $\mathfrak{M}_{k}$. Since $\left(\Re, g \mathfrak{M}_{k}\right) \approx\left(S^{3} \times R^{n-3}, \mathfrak{M}_{k}\right) \approx\left(S^{3} \times R^{n-3}, S^{3} \times B(1)\right)$, it follows that $\Re$ is homeomorphic to $g \mathfrak{M}_{k}$ with a half-open collar added on the boundary. Hence the PL structure of $g \mathfrak{M}_{k}$ extends to all of $\Re$. Let $\Re^{*}$ be the resultant PL manifold. Then $g \mathfrak{M}_{k} \mid \operatorname{Int} g M_{k}$ and $g M_{k} \mid \operatorname{Int}\left(g \mathfrak{M}_{k-1}\right)$ are PL collars in $\mathfrak{R}^{*}$. So there is a PL ambient isotopy of $\Re^{*}$ taking $g \mathfrak{M}_{k}$ to $g \mathfrak{M}_{k-1}=\mathfrak{N}_{k-1}$ and taking $g M_{k}$ to $N_{k-1}$ with a collar added on its boundary. This may be followed by a PL isotopy of $\Re$ taking $\mathfrak{N}_{k-1}$ onto $\mathfrak{N}_{k}$ and the collared $N_{k-1}$ onto $N_{k}$. Both of these isotopies can be fixed on $g M_{k-1}=N_{k-1}$. Let $h_{k, t}$ be $h_{k-1 . t}$ followed by the composition of these two isotopies appropriately reparametrized. Conditions (a), (b), and (c) are easily checked.

Define $h_{t}: S^{3} \times R^{n-3} \rightarrow \Re$ for $t \geqq 0$ by $h_{t}=h_{[t] . t}$ where $[t]$ is the greatest integer $\leqq t . h(x, t)=h_{t}(x)$ is easily seen to be continuous. Define $h_{\infty}(x)=$ $\lim _{t \rightarrow \infty} \bar{h}_{t}(x)$.

Claim 2. $h_{\infty}$ is a well-defined PL homeomorphism. Let $x \in M_{k}, t \geqq k+1$, $m=[t]$. Then $x \in M_{m-1}$ and $h_{t}(x)=h_{m, t}(x)=h_{m-1, m}(x)$ by (c). If $m>k+1$, then $m-1 \geqq k+1, x \in M_{m-2}$ and $h_{m-1 . m}(x)=h_{m-2 . m-1}(x)$. Proceeding in 
this way, $h_{t}(x)=h_{k, k+1}(x)$ for $t \geqq k+1$, i.e., $h_{\infty}=h_{k, k+1}$ on $M_{k}$. This establishes Claim 2 and shows that $h$ is continuous at $(x, \infty)$.

To finish the construction of the counterexample, set

$$
K_{t}(x)=h\left(x, \tan \frac{1}{2} \pi t\right) \text {. }
$$

Proof of Claim 1. Let $N$ be a PL regular neighborhood of $\Sigma$ in $\Re$. Pick $p>1$ such that $C \subset S^{3} \times$ Int $B(p)$. Let $U=\operatorname{Int} N$,

$$
V=\Re \backslash\left[S^{3} \times B(p)\right]=S^{3} \times\left[R^{n-3} \backslash B(p)\right] .
$$

Claim 3. $\pi_{*}(\Re, U)=0$. Via $\left(\alpha_{1}\right)_{*}, \pi_{*}(\Re, \Sigma) \cong \pi_{*}\left(\Re, S^{3} \times 0\right)=0$. Also $\pi_{*}(N, \Sigma)=0$. Hence from the homotopy sequence of $(\Re, N, \Sigma)$, $\pi_{*}(\Re, N)=0$. Clearly, $\pi_{*}(N, U)=0$. So from the homotopy sequence of $(\Re, N, U), \pi_{*}(\Re, U)=0$.

Claim 4. $\pi_{*}(\Re \mid \Sigma, V)=0 . \pi_{*}(\Re \mid \Sigma, V) \cong \pi_{*}\left(\Re \backslash\left(S^{3} \times 0\right), V\right)=0$ via $\left(\alpha_{1}\right)_{*}$ since $\alpha$ is fixed on $V$.

Claims 3 and 4 are the necessary connectivity assumptions to apply a PL engulfing argument as in [4, pp. 169-170] to find a PL homeomorphism $k$ of $\Re$ fixed on $\Sigma$ such that $C \subset k N$ where $k N$ is still a regular neighborhood of $\Sigma$. Claim 1 is thus established.

4. The necessity of the codimension-three hypothesis. By [5] for every integer $k \geqq 0$ there are PL manifolds $K_{k} \subset U_{k} \subset$ Int $V_{k}$ where $U_{k}$ is a PL regular neighborhood of $K_{k}$ in $V_{k}$ and the codimension of $K_{k}$ in $V_{k}$ is two. Proposition 2 of [5] gives a homeomorphsim $h:\left(V_{k}, K_{k}\right) \rightarrow\left(V_{0}, K_{0}\right)$. Then $h\left(U_{k}\right)$ is a topological regular neighborhood of $K_{0}$. If the Theorem were true in codimension two, $h$ could be changed via an isotopy so that $h\left(U_{k}\right)=U_{0}$. If $N_{k}=V_{k} \mid$ Int $U_{k}$, then $h\left(N_{k}\right) \approx \partial U_{0} \times I$. Since Whitehead torsion is a topological invariant, it follows from (22.7) of [3] that $h_{*} \tau\left(N_{k}, \partial U_{k}\right)=\tau\left(\partial U_{0} \times I, \partial U_{0} \times 0\right)=0$. Since $h_{*}$ is an isomorphism, $\tau\left(N_{k}, \partial U_{k}\right)=0$, which contradicts Assertion 2 of [5].

\section{REFERENCES}

1. M. A. Armstrong, Collars and concordances of topological manifolds, Comment. Math. Helv. 45 (1970), 119-128. MR 42 \#1128.

2. T. Chapman, The topological invariance of Whitehead torsion, Amer. J. Math. (to appear).

3. M. M. Cohen, A course in simple-homotopy theory, Graduate Texts in Math. 10, Springer-Verlag, New York (to appear).

4. J. F. P. Hudson, Piecewise linear topology, Univ. of Chicago Lecture Notes prepared with the assistance of J. L. Shaneson and J. Lees, Benjamin, New York, 1969. MR 40 \#2094.

5. M. Kato, Regular neighborhoods are not topologically invariant, Bull. Amer. Math. Soc. 74 (1968), 988-991. MR 37 \#804. 
6. R. C. Kirby and L. C. Siebenmann, On the triangulation of manifolds and the Hauptvermutung, Bull. Amer. Math. Soc. 75 (1969), 742-749. MR 39 \#3500.

7. - Some theorems on topological manifolds, Manifolds-Amsterdam, 1970 (Proc. Nuffic Summer School), Lecture Notes in Math., vol. 197, Springer, Berlin, 1971, pp. 1-7. MR 44 \#1037.

8. K. W. Kwun, Uniqueness of the open cone neighborhood, Proc. Amer. Math. Soc. 15 (1964) 476-479. MR 28 \#4527.

9. K. W. Kwun and F. Raymond, Mapping cylinder neighborhoods, Michigan Math. J. 10 (1963), 353-357. MR 27 \#6248.

10. R. T. Miller, Close isotopies on piecewise linear manifolds, Trans. Amer. Math. Soc. 151 (1970), 597-628. MR 42 \#1127.

11. J. Milnor, Whitehead torsion, Bull. Amer. Math. Soc. 72 (1966), 358-426. MR 33 \#4922.

Department of Mathematics, University of Illinois at Chicago Circle, Chicago, IllinoIs 60680 